Background: Vasculitis in patients with sarcoidosis is rare and can affect blood vessels of any size. Limited information describing this association is available. Objectives: To describe a large, single-institution case-series of patients with sarcoidosis and vasculitis.

Methods: A single-institution retrospective study was performed reviewing all patients with a diagnosis code for sarcoidosis and vasculitis between January 1, 1998 and December 31, 2019. Medical records were individually reviewed, and data regarding diagnosis, treatment, and outcomes were abstracted. Sarcoidosis was diagnosed based on histologic, radiographic, and clinical data with exclusion of alternative diagnoses. Diagnosis for small vessel vasculitis (SVV) required biopsy confirmation. Patients with large or medium-vessel vasculitis (L/ MVV) required biopsy and/or arterial imaging. Comparison between patients presenting with L/MVV versus patients with only SVV was performed.

Results: Seventeen patients were identified during the study period. Nine patients (56\% female) had L/MVV and 8 (50\% female) had SVV. Sarcoidosis preceded the diagnosis of vasculitis in 4 (44\%) patients in the L/MVV group compared to $3(38 \%)$ patients in the SVV group. Mean \pm SD age at sarcoidosis diagnosis was $53.2 \pm 17.8$ and $51.9 \pm 11.4$ years and mean $\pm S D$ age at vasculitis diagnosis was $57.4 \pm 19.6$ and $59.0 \pm 13.4$ years in L/MVV and SVV groups, respectively. Number of organ systems involved by sarcoidosis was similar [median (IQR) 3 $(1,4)$ L/MVV vs. $2.5(1.75,3.25)$ SVV]. Sarcoid organ involvement and vasculitis vessel distributions are noted in Table 1. Mean length of follow-up was $11.5 \pm 12.8$ years in L/MVV and $13.1 \pm 14.3$ years in SVV. All patients were treated with glucocorticoids for vasculitis. More patients with L/MVV were treated with at least one DMARD/biologic $(8 / 9,89 \%)$ compared to SVV $(3 / 8,38 \%, p=0.05)$. Complete response to therapy for vasculitis was observed in 8/9L/MVV and 7/8 in SVV. Four patients in SVV group were able to stop all immunosuppression but only one patient with L/MVV was off all therapy at last follow-up.

\begin{tabular}{|c|c|c|c|c|c|}
\hline Case & Sex & $\begin{array}{l}\text { Age Sarcoid } \\
\text { /Vasculitis } \\
\text { Dx, yr }\end{array}$ & $\begin{array}{l}\text { WASOG Organ } \\
\text { Involvement }{ }^{\star}\end{array}$ & Vessel size & $\begin{array}{l}\text { Arterial involvement } \\
\text { on imaging } ¥ ¥ \text { or biopsy }{ }^{\star \star}\end{array}$ \\
\hline 1 & M & $44 / 44$ & B-J, ET-LN, Sk & LVV, MVV & $\begin{array}{r}\text { AA (e), Ant Tib (s), Ax (s), Br } \\
\text { (o), V (s), Ca (s), CF (b), I } \\
\text { (b), Innom (b), Mes (b, o, s) } \\
\text { Per (b), Pop (a), Re (b), SF } \\
\text { (b), Sub (o, pa, wt), TA (a) }\end{array}$ \\
\hline 2 & $\mathrm{~F}$ & $66 / 68$ & $\begin{array}{c}\text { BM, ET-LN, Li, Lu, } \\
\text { NS, S }\end{array}$ & LVV & TA (hm) \\
\hline 3 & $\mathrm{~F}$ & $55 / 55$ & Lu, Sp & MVV & IMA (a, s, o, wt), Sp (a) \\
\hline 4 & M & $81 / 80$ & Lu & LVV & $\begin{array}{l}\text { AA }(\mathrm{hm}), \text { Car }(\mathrm{hm}), \text { Innom } \\
(\mathrm{hm}), \text { Sub }(\mathrm{hm}), \mathrm{TA}(\mathrm{hm})\end{array}$ \\
\hline 5 & $\mathrm{~F}$ & $72 / 72$ & Eye, NS, Lu & LVV, MVV & $\begin{array}{c}\text { ACA (b), ICA (b, en, s), MCA } \\
\text { (b), SMA (wt) }\end{array}$ \\
\hline 6 & M & $43 / 45$ & Lu & LVV, MVV & $\begin{array}{c}\operatorname{Br}(o, s), C e(s), \text { SMA (s), Sp } \\
(\mathrm{s}), \text { TA (wt) }\end{array}$ \\
\hline 7 & $\mathrm{~F}$ & $53 / 54$ & B-J, Eye, Lu, Sk & MVV & Skin - medium vessel ${ }^{\star \star}$ \\
\hline 8 & M & $24 / 20$ & Eye, Li, Lu, Sp & MVV & Hep (a), Sp (a) \\
\hline 9 & $\mathrm{~F}$ & $40 / 79$ & Lu & LVV & Temporal artery** \\
\hline 10 & M & $55 / 57$ & Li, Lu, NS & SVV & Sural nerve ${ }^{\star \star}$ \\
\hline 11 & $\mathrm{~F}$ & $53 / 50$ & ET-LN, Lu, Sk & SVV & Skin ${ }^{\star *}$ \\
\hline 12 & M & $60 / 62$ & Lu & SVV & Skin $^{\star \star}$ \\
\hline 13 & $\mathrm{~F}$ & $67 / 82$ & ET-LN, Lu & SVV & Skin ${ }^{\star \star}$ \\
\hline 14 & $\mathrm{~F}$ & $52 / 56$ & Eye, Lu, NS, Sk & SVV & Skin ${ }^{* *}$ \\
\hline 15 & $M$ & $39 / 37$ & Eye, Lu, NS, Sk & SVV & Skin $^{\star *}$ \\
\hline 16 & $\mathrm{~F}$ & $57 / 56$ & Lu, Sk & SVV & Skin ${ }^{\star *}$ \\
\hline 17 & M & $32 / 71$ & $\mathrm{Lu}$ & SVV & Skin $^{\star \star}$ \\
\hline
\end{tabular}

*WASOG Organ Involvement: B-J, bone-joint; BM, bone marrow; ET-LN, extra-thoracic lymph node; Li, liver; Lu, lung; NS, nervous system; Sk, skin; Sp, spleen

† a, aneurysm; b, beaded irregularities; e, ectasia; en, enhancement; hm, hypermetabolism (on positive emission tomography); o, occlusion; pa, pseudoaneurysm; s, stenosis; wt, wall thickening

$¥ \mathrm{AA}$, abdominal aorta; $\mathrm{ACA}$, anterior cerebral artery; Ant Tib, anterior tibial; $\mathrm{Ax}$, axial; $\mathrm{Br}$, brachial; Ca, carotid; Ce, celiac; CF, common femoral; Hep, hepatic; I, iliac; ICA, internal carotid artery; IMA, inferior mesenteric artery; Innom, innominate; MCA, middle cerebral artery; Mes, mesenteric branch; PCA, posterior cerebral artery; Per, peroneal; Pop, popliteal; Re, renal; SF, superficial femoral; Sp, splenic; Sub, subclavian; TA, thoracic aorta; V, vertebral

Conclusion: Vasculitis in patients with sarcoidosis is uncommon. Variability exists in the vessels involved and the treatments utilized. Overall this series observed favorable outcomes with a high percentage achieving complete response, regardless of vessel size affected.

Disclosure of Interests: None declared

DOI: 10.1136/annrheumdis-2021-eular.1146

\section{POS0802 18F-FLUORODEOXYGLUCOSE POSITRON EMISSION TOMOGRAPHY AS A PREDICTOR OF ANGIOGRAPHIC PROGRESSION OF DISEASE IN LARGE-VESSEL VASCULITIS}

K. A. Quinn ${ }^{1}$, M. Ahlman ${ }^{2}$, H. Alessi ${ }^{1}$, A. Malayeri', J. Marko², E. Novakovich', P. Grayson ${ }^{1} .{ }^{1}$ National Institutes of Health, NIAMS, Bethesda, United States of
America; ${ }^{2}$ National Institutes of Health, Clinical Center, Radiology and Imaging Sciences, Bethesda, United States of America

Background: Giant cell arteritis (GCA) and Takayasu's arteritis (TAK) are the two main forms of large-vessel vasculitis (LVV). Although angiography is essential to detect vascular disease in patients with LVV, there is limited prospective data characterizing change in arterial lesions over time, and factors that predict angiographic change remain unknown.

Objectives: The objectives of this study were to: 1) describe longitudinal change in angiographic studies in patients with GCA and TAK and 2) determine whether FDG-PET activity predicts angiographic progression of disease.

Methods: Patients with GCA or TAK were recruited into a prospective, observational cohort. All patients underwent baseline magnetic resonance (MR) or computed tomography (CT) angiography and a follow-up study (same modality) $\geq 6$ months after baseline per a standardized imaging protocol. For patients who had multiple angiograms, the baseline and most recent images were compared. Arterial lesions, defined as stenosis, occlusion, or aneurysm, were evaluated by visual inspection in 4 segments of the aorta and 13 branch arteries by a single reader blinded to clinical status. On follow up angiography, the development of new lesions in these same territories was recorded, and existing lesions were characterized as improved, worsened, or unchanged by visual inspection, with confirmation by an independent reader.

All patients underwent FDG-PET on the same date as angiography. Qualitative assessment of FDG uptake was performed in each corresponding arterial territory evaluated by angiography. Active vasculitis was defined as greater FDG uptake in the arterial wall compared to the liver by visual inspection.

Results: At the baseline visit, there were 248 arterial lesions (21\%) out of 1162 arterial territories evaluated from 70 patients with LVV $(T A K=38 ; G C A=32)$. Baseline characteristics were as follows: Age [TAK=29.5 years (18.4-39.5), GCA=69.6 years (60.7-75.5)], Female gender [TAK $=30$ patients $(79 \%), \mathrm{GCA}=23$ patients $(72 \%)]$, Disease duration $[\mathrm{TAK}=2.2$ years $(0.6-5.5), \mathrm{GCA}=0.7$ years $(0.1-2.6)$ ], Active clinical disease [TAK=17 patients $(45 \%), \mathrm{GCA}=20$ patients $(63 \%)$ ].

Over 1.6 years $(1.0-2.7)$ of median follow-up, no angiographic change was observed in 1,132 (97\%) arterial territories. New lesions developed in 8 arterial territories, exclusively in 5 patients with TAK. Arterial lesions improved in 16 territories $(\mathrm{GCA}=7, \mathrm{TAK}=9)$ and worsened in 6 territories $(\mathrm{GCA}=1, \mathrm{TAK}=5)$. Patients with angiographic improvement were initially imaged earlier in the disease course compared to patients with new/worsening lesions (median 1.1 vs 16.4 months, $\mathrm{p}=0.09$ ). Patients with angiographic improvement had significantly lower acute phase reactants at follow-up compared to patients with new/worsening arterial lesions [median ESR 3.0 (2.0-15.0) vs. 27.0 (7.3-39) mm/h, p<0.01; median CRP $0.7(0.3-1.4)$ vs. $6.1(3.1-19.6) \mathrm{mg} / \mathrm{L}, \mathrm{p}<0.01]$. Seventy-nine percent of patients with new/worsening arterial lesions had received increased treatment over the follow-up interval compared to $100 \%$ patients with improved arterial lesions, $p=0.09$. FDG-PET activity was evaluated in 1091/1162 (94\%) of corresponding arterial territories. PET activity in an arterial territory at baseline was significantly associated with change in that arterial territory (either new/worsening or improvement) on follow-up angiography $(p<0.01)$ (FIGURE 1). PET activity had a sensitivity of $80 \%$ and specificity of $74 \%$ for predicting change in arterial lesions. Most arterial territories without PET activity at baseline remained unchanged over time by angiography, yielding a negative predictive value of 99\%. (FIGURE 1).

Conclusion: Development of new arterial lesions is infrequent in LVV. Change in arterial lesions is dynamic, and improvement can occur. FDG-PET activity predicts change in angiographic lesions, and lack of PET activity is strongly associated with stable angiographic disease. These data may inform guideline recommendations for imaging monitoring in LVV.

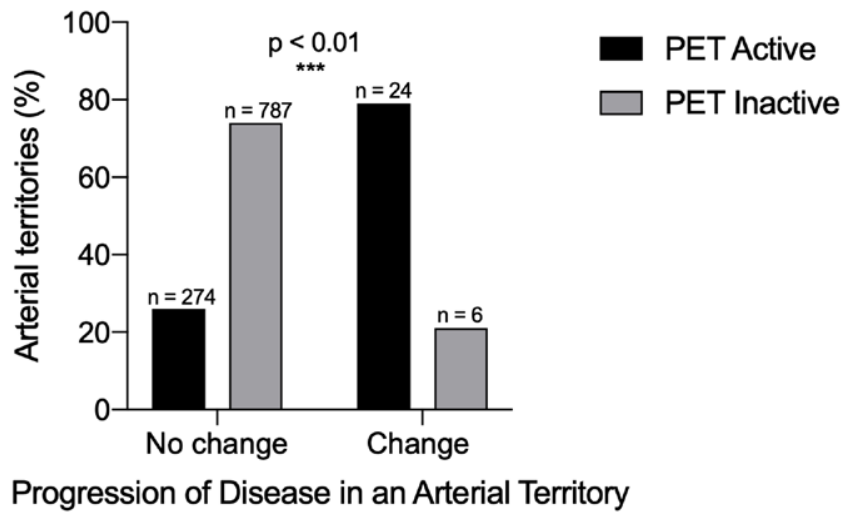

Figure 1.

Disclosure of Interests: None declared

DOI: 10.1136/annrheumdis-2021-eular.1278 